\title{
Many-Body Resonance in a Correlated Topological Kagome Antiferromagnet
}

\author{
Songtian Sonia Zhang, ${ }^{1, *}$ Jia-Xin Yin $\odot,{ }^{1, *}, \dagger$ Muhammad Ikhlas, ${ }^{2}$ Hung-Ju Tien $\odot,{ }^{3}$ Rui Wang, ${ }^{4}$ Nana Shumiya, \\ Guoqing Chang $\odot,{ }^{1}$ Stepan S. Tsirkin $\odot,{ }^{5}$ Youguo Shi ${ }^{6,7}$ Changjiang Yi, ${ }^{6,7}$ Zurab Guguchia $\odot,{ }^{8}$ Hang Li, ${ }^{6}$ Wenhong Wang, ${ }^{6}$ \\ Tay-Rong Chang, ${ }^{3}$ Ziqiang Wang, ${ }^{9}$ Yi-feng Yang, ${ }^{6,7}$ Titus Neupert, ${ }^{5}$ Satoru Nakatsuji, ${ }^{2,10,11}$ and M. Zahid Hasan ${ }^{1,12,:}$ \\ ${ }^{1}$ Laboratory for Topological Quantum Matter and Advanced Spectroscopy, Department of Physics, Princeton University, \\ Princeton 08544, New Jersey, USA \\ ${ }^{2}$ Institute for Solid State Physics, University of Tokyo, Kashiwa 277-8581, Japan \\ ${ }^{3}$ Department of Physics, National Cheng Kung University, Tainan 701, Taiwan \\ ${ }^{4}$ National Laboratory of Solid State Microstructures and Department of Physics, Nanjing University, \\ Nanjing 210093, China \\ ${ }^{5}$ Department of Physics, University of Zurich, Zurich 8057, Switzerland \\ ${ }^{6}$ Beijing National Laboratory for Condensed Matter Physics and Institute of Physics, Chinese Academy of Sciences, \\ Beijing 100190, China \\ ${ }^{7}$ University of Chinese Academy of Sciences, Beijing 100049, China \\ ${ }^{8}$ Laboratory for Muon Spin Spectroscopy, Paul Scherrer Institute, Villigen PSI CH-5232, Switzerland \\ ${ }^{9}$ Department of Physics, Boston College, Chestnut Hill, Massachusetts 02467, USA \\ ${ }^{10}$ Department of Physics, University of Tokyo, Hongo, Bunkyo-ku, Tokyo 113-0033, Japan \\ ${ }^{11}$ CREST, Japan Science and Technology Agency (JST), 4-1-8 Honcho Kawaguchi, Saitama 332-0012, Japan \\ ${ }^{12}$ Material Sciences Division, Lawrence Berkeley National Laboratory, Berkeley, California 94720, USA
}

(Received 29 March 2020; accepted 26 June 2020; published 21 July 2020)

\begin{abstract}
We use scanning tunneling microscopy to elucidate the atomically resolved electronic structure in the strongly correlated kagome Weyl antiferromagnet $\mathrm{Mn}_{3} \mathrm{Sn}$. In stark contrast to its broad single-particle electronic structure, we observe a pronounced resonance with a Fano line shape at the Fermi level resembling the many-body Kondo resonance. We find that this resonance does not arise from the step edges or atomic impurities but the intrinsic kagome lattice. Moreover, the resonance is robust against the perturbation of a vector magnetic field, but broadens substantially with increasing temperature, signaling strongly interacting physics. We show that this resonance can be understood as the result of geometrical frustration and strong correlation based on the kagome lattice Hubbard model. Our results point to the emergent many-body resonance behavior in a topological kagome magnet.
\end{abstract}

DOI: 10.1103/PhysRevLett.125.046401

Studying the effects of correlation and topology in quantum materials is emerging as one of the central themes in condensed matter physics [1]. In electron systems with strong Coulomb interaction, they often exhibit exotic electronic and magnetic properties that cannot be sufficiently accounted for by the noninteracting properties of their individual constituents. As such, the realization of these emergent properties in topological materials can lead to unpredicted manifestations of their many-body physics. Recently, a series of correlated kagome magnets have been observed to have anomalous transport response, correlated topological electronic structure, and giant spin-orbit tunability [2-16]. Among these, $\mathrm{Mn}_{3} \mathrm{Sn}$ stands out due to its antiferromagnetism and the absence of any nonkagome layers. It is also one of the rare antiferromagnets that exhibits large anomalous Hall and Nernst effects, arising from the Berry curvature due to gapped magnetic nodal lines leading to Weyl fermions [2,3]. In addition to its large Berry curvature, photoemissions experiments have shown a broad single-particle electronic structure with a band renormalization factor as large as five [4]. Therefore, this combination of kagome lattice, topological bands, and strong correlation in $\mathrm{Mn}_{3} \mathrm{Sn}$ lends itself to be a fascinating platform for studying the strongly correlated topological kagome magnet.

$\mathrm{Mn}_{3}$ Sn crystallizes in space group $P 6_{3} / m m c$ with the lattice constant $a=b=5.7 \AA$, where each layer consists of a kagome lattice made of $\mathrm{Mn}$ atoms stuffed with $\mathrm{Sn}$ atoms [Fig. 1(a)], with $A B$ stacking of the equivalent layers [Fig. 1(b)]. A first-principles calculation of the bulk band structure [Fig. 1(c)] finds several flatbands and Weyl fermions [3,4]. Because of strong interplane coupling, the as-cleaved surface does not exhibit an atomic lattice structure [5,12]. To prepare atomic surfaces, we first cleave the sample and then anneal it at $1100 \mathrm{~K}$ for one hour. With this in situ annealing process, we can measure large clean flat areas with terraces larger than $300 \mathrm{~nm}$ in size [Fig. 1(d)]. We directly visualize the $\mathrm{Mn}_{3} \mathrm{Sn}$ atomic surface at $4.6 \mathrm{~K}$ with hexagonal symmetry and expected lattice constant, as shown in Fig. 1(e). A comparison to simulation 



(d) In situ annealed

(e)

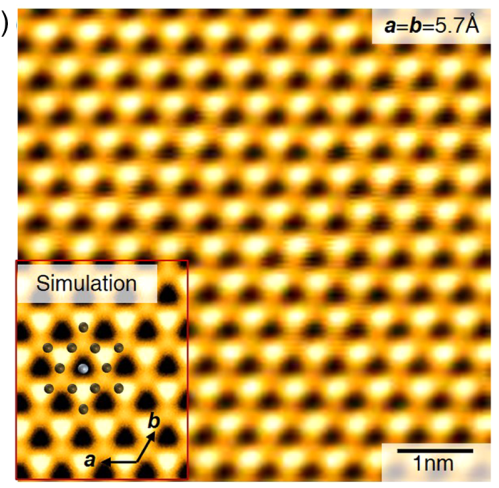

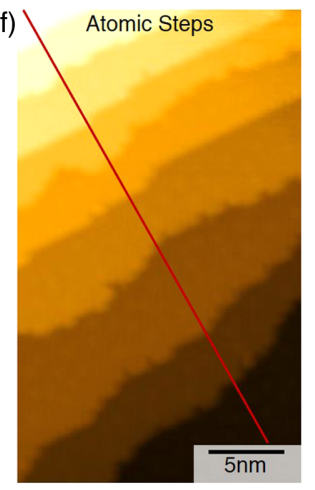
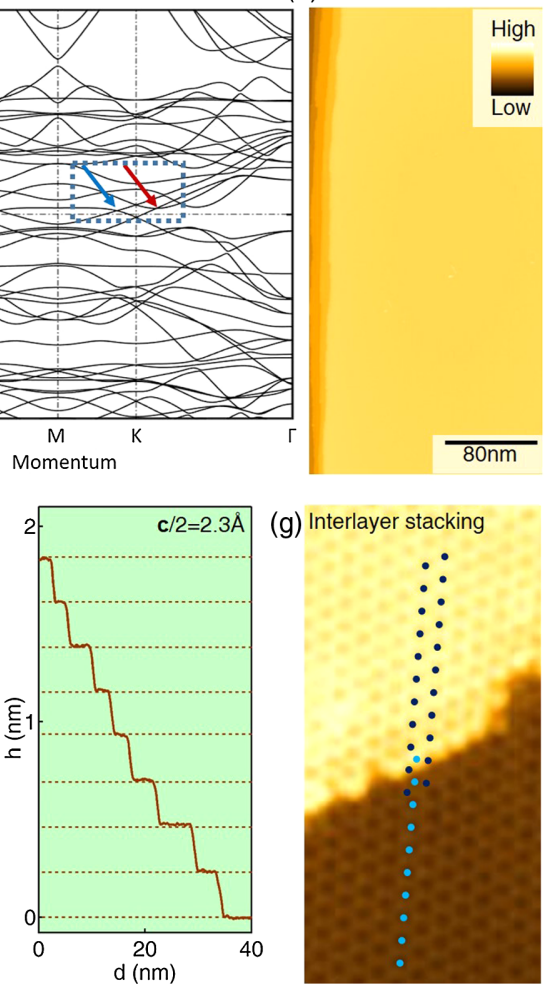

FIG. 1. (a) Crystal structure of the $\mathrm{Mn}_{3}$ Sn kagome lattice. (b) $c$-axis $A B$ stacking of the kagome lattice from side view and top view, respectively. (c) Normalized bulk band structure of $\mathrm{Mn}_{3} \mathrm{Sn}$ in the conformal Brillouin zone showing low energy flatbands (black arrows) and Weyl nodes with two examples indicated by red and blue arrows. (d),(e) STM topographic images at different scales of Mn ${ }_{3} \mathrm{Sn}_{\text {after }}$ annealing the cleaved surface at $1100 \mathrm{~K}$ for $1 \mathrm{~h}$, showing a large atomic flat surface showing the lattice with hexagonal symmetry. (e) inset: First-principles simulation of the image overlaid with atoms illustrations. (f) STM topographic image across multiple steps of single layers, whose line profile is shown on the right. (g) Stacking alignment of two layers across a step, with the black and blue dots denoting $\mathrm{Sn}$ atoms of the upper and lower layers, respectively. All topographic data were taken at $T=4.6 \mathrm{~K}, V=-50 \mathrm{mV}, I=50 \mathrm{pA}$.

finds reasonable agreement [Fig. 1(e), inset], similar to the kagome surfaces in $\mathrm{Fe}_{3} \mathrm{Sn}_{2}[5,13]$ and $\mathrm{Co}_{3} \mathrm{Sn}_{2} \mathrm{~S}_{2}[10,15]$. An analysis of a line cut taken across several single atomic steps finds each step to be approximately $2.3 \AA$, consistent with its half $c$-axis unit [Fig. 1(f)]. Further analysis across a single atomic step reveals the interlayer alignment of the kagome lattice, consistent with the $A B$ stacking of the bulk crystal structure [Fig. 1(g)].

Having visualized the atomic kagome surface of $\mathrm{Mn}_{3} \mathrm{Sn}$, we now investigate its low-energy electronic structure. Measuring the $d I / d V$ spectrum along a line of $50 \mathrm{~nm}$, far from any step edge, we find a consistent resonance feature at $E_{F}$ with a line shape asymmetry [Fig. 2(a)]. Near the atomic step edge, we find that the resonance is strongly suppressed [Fig. 2(b) and its inset], demonstrating that the resonance is not from the breaking bonding induced scattering from the step edge. As this peak is observed at every position on the kagome surface, this resonance state is not related to an isolated impurity but is a feature of the kagome lattice. This point is further supported in Fig. 2(c), that the resonance is slightly suppressed by the isolated atomic impurity. The spectral asymmetry of this resonance leads us to consider the Fano equation $F(E) \approx\left[(q+E / \Gamma)^{2}\right] /\left[1+(E / \Gamma)^{2}\right]$, where $q$ is the quality factor that quantifies the coupling of the tip to the discrete state and $\Gamma$ is the resonance width. The spatially averaged $d I / d V$ spectrum can indeed be fitted by $A^{*} F(E)+B$ as shown in Fig. 2(d), where $A$ and $B$ are the additional adjustment parameters. It can also be seen that there is a nonzero background, indicative that not all states are associated with the resonance. $\Gamma=3.9 \mathrm{meV}$ is the characteristic energy scale of the resonance. If we associate this state with the Kondo lattice resonance, the estimated Kondo temperature would amount to $T_{\mathrm{K}}=\Gamma / 1.4 k_{B}=32 \mathrm{~K}$.

A magnetic field dependent measurement finds that despite some weak broadening the resonance peak does not split or shift under an out-of-plane $4 \mathrm{~T}$ field or in-plane $2 \mathrm{~T}$ field relative to the unperturbed resonance state [Fig. 3(a) orange, brown, and blue curves, respectively]. In reference to tunneling experiments in $\mathrm{YbRh}_{2} \mathrm{Si}_{2}$ and $\mathrm{SmB}_{6}$ among others $[6,7,17]$ where a Kondo resonance with a Fano line shape was observed, the resonance was also not split by a strong magnetic field. In contrast to the weak field response, we observe a strong temperature 

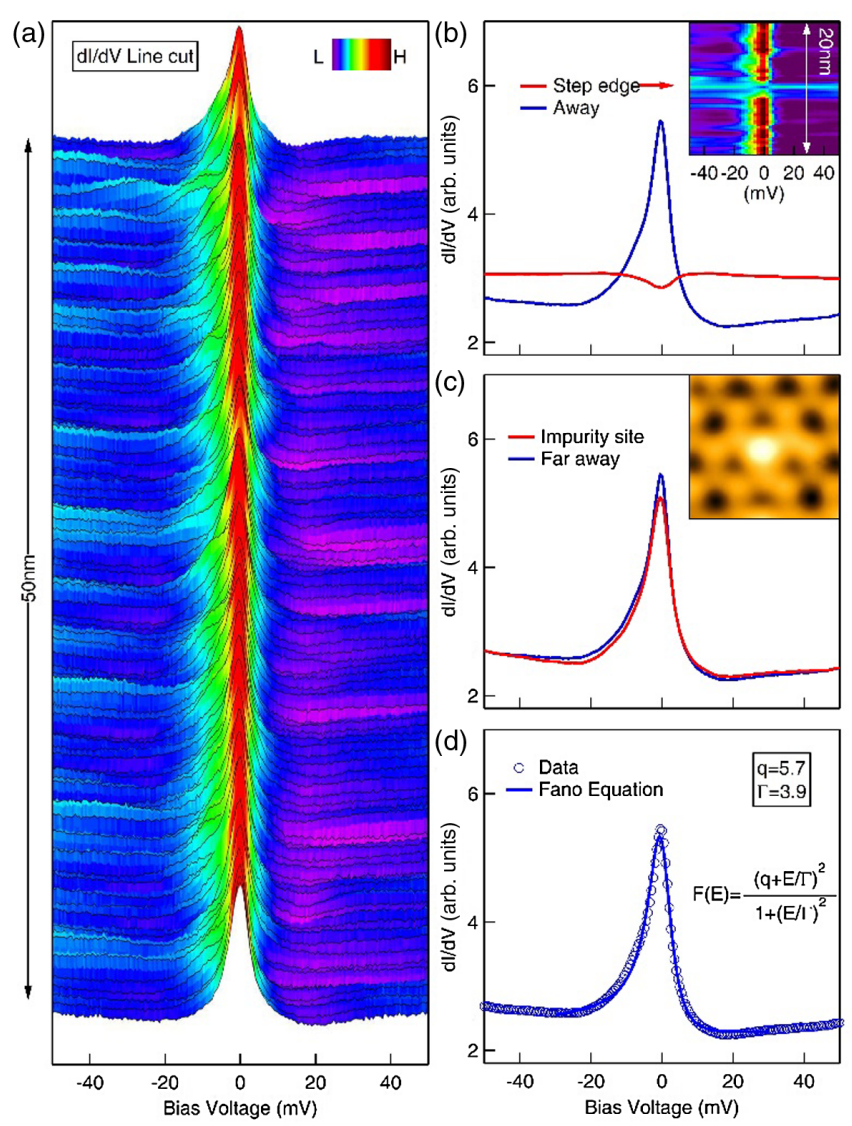

FIG. 2. (a) $d I / d V$ line cut across $50 \mathrm{~nm}$, taken far from any step edge, showing a peak at $E_{F}$. Every fifth curve is marked by a solid black line for clarity. (b) Perturbation of a step edge on the Fermi level resonance. The inset shows the line cut spectra taken across a single step edge, whose position is marked by the arrow. (c) Perturbation of the atomic impurity on the Fermi level resonance. The inset shows the topographic image of the impurity. (d) Spatially averaged $d I / d V$ spectrum (open circles). The solid line is a fit to Fano line shape function, $F(E)=\left[(q+E / \Gamma)^{2}\right] /\left[1+(E / \Gamma)^{2}\right]$.

dependence of the resonance. Our temperature-dependent measurement finds that the resonance peak is substantially suppressed and broadens with increasing temperature [Fig. 3(b), solid lines]. In tunneling experiments, the $d I / d V$ spectra measure the convolution of the DOS and the derivative of the Fermi Dirac distribution function. As such, we also plot the temperature convolution of the spectrum taken at our lowest temperature $(T=4.6 \mathrm{~K})$ for each temperature (dotted lines) for comparison. We see that the actual data show a stronger temperature broadening effect than the convoluted curves, indicating an interaction driven resonance with intrinsic temperature dependence. Next, we fit the experimental data with the thermally convoluted Fano function in Fig. 3(c). During the fitting, we find that although $q$ shows little variation, $\Gamma$ increases substantially with $T$, which is associated with the intrinsic thermal broadening of the DOS peak. The spatially
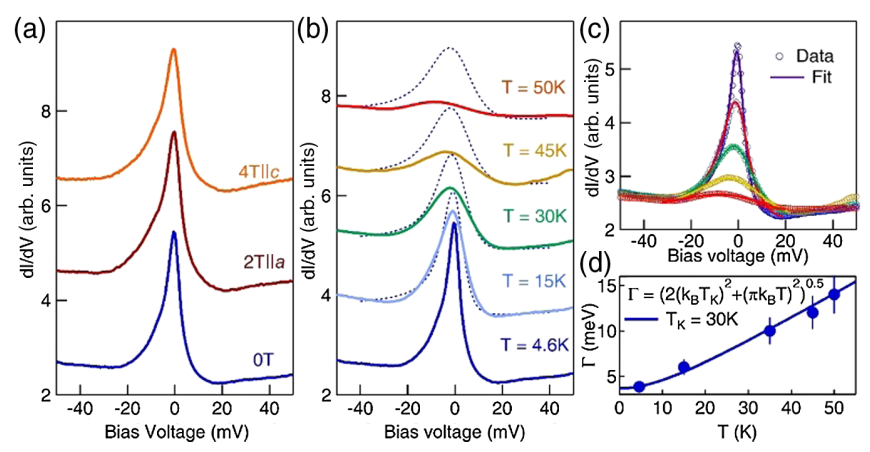

FIG. 3. (a) $d I / d V$ taken at $0 \mathrm{~T}$ (blue curve), $2 \mathrm{~T}$ along $a$ axis (brown curve), and $4 \mathrm{~T}$ along $c$ axis (yellow curve). (b) Temperature dependence of $d I / d V$ with spectra offset for clarity. The dotted lines are numerically calculated spectra by convoluting the 4.6 $\mathrm{K}$ data with the derivative of Fermi-Dirac distribution function at respective temperatures. (c) Fit of the temperaturedependent spectra by thermally convoluted Fano function. (d) Resonance width $\Gamma$ plotted against temperature, giving a Kondo temperature of $T_{K}=30 \mathrm{~K}$ when fit to the Kondo model.

independent line shape, magnetic field response, and thermal broadening of this resonance all resemble the behavior of the resonance in systems that can be described by the Kondo lattice model [6,7,17-20]. Fitting $\Gamma(T)$ with the extended phenomenological expression derived initially for the single-impurity model [21-23], $\Gamma=\left[2\left(k_{B} T_{K}\right)^{2}+\left(\pi k_{B} T\right)^{2}\right]^{0.5}$, we obtain an estimated Kondo temperature of $T_{K}=30 \mathrm{~K}$, as shown in Fig. 3(d). The obtained $T_{K}$ is consistent with the energy scale of the linewidth at base temperature. In the bulk resistivity data [24], we have also observed a characteristic Kondo upturn around this $T_{K}$ determined by scanning tunneling microscopy (STM) or scanning tunneling spectroscopy, indicating its bulk origin. We can use this estimated Kondo temperature to reexamine the nonsplitting nature of the resonant state under the magnetic field. The minimum field with which the resonant state splits is related to the Kondo temperature by approximately $g \mu_{B} B \approx 0.5 k_{B} T_{K}[44,45]$. For an estimated $T_{K}=30 \mathrm{~K}$ and $g \approx 2$, a minimum field of approximately $11 \mathrm{~T}$ would be required. For the smaller fields applied in our experiment, some broadening of the resonance can be observed, but without clearly splitting.

Indeed, there are several ways in which the crystal and electronic structure of $\mathrm{Mn}_{3} \mathrm{Sn}$ can be considered analogous to systems described by the Kondo model [46]. In the Kondo model, the localized moments in a lattice are screened by the itinerant conductive sea, forming periodic singlet states [Fig. 4(a)]. In their excitation spectrum, the many-body interactions between the flatband and itinerant conduction band can manifest as a resonance with a Fano line shape at the Fermi level [Fig. 4(b)]. This behavior is mostly observed in heavy fermion systems with localized $f$ orbitals. However, the key components for the formation of such a resonance-namely the flatband and the strong 


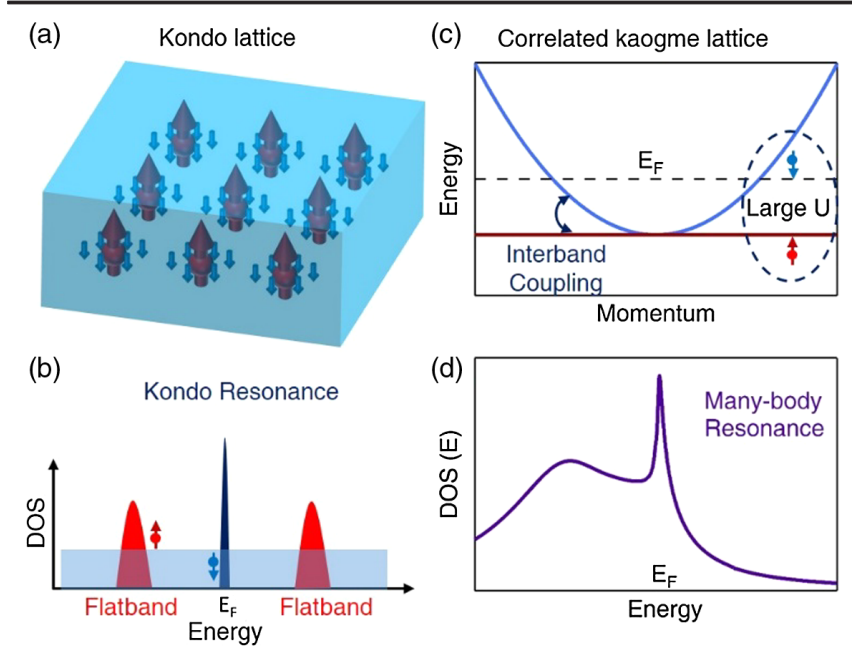

FIG. 4. (a) Schematic depicting a Kondo lattice formed by the coupling between periodic localized states (red arrows) and itinerant conduction electrons (blue arrows). (b) DOS spectrum of the Kondo lattice, where a Kondo resonance (dark blue) at $E_{F}$ is generated by the many-body coupling of the localized flatband (red) and itinerant conduction band (shaded blue). (c) Band structure for a kagome tight-binding model showing a flatband with interband coupling and a large Hubbard interaction. (d) The calculated DOS shows a weak bump arising from the kagome flatband and a many-body resonance at $E_{F}$ due to the hybridization of the localized and itinerant states.

Coulomb interactions - can, in principle, be satisfied in a strongly correlated $3 d$ kagome metal. In this case, the kagome lattice localizes the electronic wave functions in place of the heavy $f$ electrons, with the itinerant conduction sea naturally arising from its metallic state. Although the higher-energy single-particle flatband cannot be clearly resolved in this strongly correlated system due to the short quasiparticle lifetime [4], the flatband is a general feature of the kagome lattices as demonstrated in its first-principles calculations. Theoretically, we consider a set of kagome flatbands touching a dispersive band with a dominating Hubbard $U$ and an interband coupling [Fig. 4(c)]. By solving the coupled equation of motion for the Green's function derived to the third order for the kagome lattice Hubbard model [24], we show that there can be a manybody resonance at the Fermi level in the density of states [Fig. 4(d)]. While our model considers electronic frustration, we note that magnetic frustration could also introduce heavy fermions [47].

Moreover, in contrast to heavy fermion materials, $\mathrm{Mn}_{3} \mathrm{Sn}$ exhibits anomalous Hall and Nernst effects coming from Berry curvature with topological fermions and Fermi arcs $[2,4,11]$, and the interplay between frustrated magnetism, Berry phase, and many-body effects within this material has the potential to open new research directions. The achievement of a large atomic flat surface in this material by our methodology also indicates the possibility of engineering such stoichiometric materials down to

atomically thin layers to realize the quantized anomalous effect toward the realization of high-temperature interacting dissipationless modes. Finally, it has not escaped our attention that previous tunneling data directly into the kagome layer of $\mathrm{Fe}_{3} \mathrm{Sn}_{2}$ and $\mathrm{Co}_{3} \mathrm{Sn}_{2} \mathrm{~S}_{2}$ all exhibit an anomalous zero-bias peak $[5,10,13,15]$; therefore, this many-body resonance phenomenon may be ubiquitous in this family of strongly correlated kagome metals.

Experimental and theoretical work at Princeton University was supported by the Gordon and Betty Moore Foundation (Grants No. GBMF4547 and No. GBMF9461/Hasan). Sample characterization was supported by the U.S. Department of Energy (U.S. DOE) under the Basic Energy Sciences program (Grant No. DOE/BES DE-FG02-05ER46200). S.S.T. and T.N. acknowledge support from the European Union's Horizon 2020 research and innovation programme (ERC-StG- Neupert-757867PARATOP) and NCRR Marvel. T.-R.C. acknowledges support from the MOST Young Scholar Fellowship (MOST Grant for the Columbus Program No. 107-2636M-006-004-), the National Cheng Kung University, Taiwan, and the National Center for Theoretical Sciences (NCTS), Taiwan. Z.W. is supported by the U.S. Department of Energy, Basic Energy Sciences Grant No. DE-FG0299ER 45747. This work is partially supported by CREST (JPMJCR18T3), Japan Science and Technology Agency, by Grants-in-Aids for Scientific Research on Innovative Areas (15H05882 and 15H05883) from the Ministry of Education, Culture, Sports, Science, and Technology of Japan, and by Grants-in-Aid for Scientific Research (19H00650) from the Japanese Society for the Promotion of Science (JSPS). M.Z.H. acknowledges support from Lawrence Berkeley National Laboratory and the Miller Institute of Basic Research in Science at the University of California, Berkeley in the form of a Visiting Miller Professorship. Y. S. acknowledges support from the National Key Research and Development Program of China (No. 2017YFA0302901).

*These authors contributed equally to this work.

Corresponding author.

jiaxiny@princeton.edu

Corresponding author. mzhasan@princeton.edu

[1] B. Keimer and J.E. Moore, The physics of quantum materials, Nat. Phys. 13, 1045 (2017).

[2] S. Nakatsuji, N. Kiyohara, and T. Higo, Large anomalous Hall effect in a non-collinear antiferromagnet at room temperature, Nature (London) 527, 212 (2015).

[3] H. Yang et al., Topological Weyl semimetals in the chiral antiferromagnetic materials $\mathrm{Mn}_{3} \mathrm{Ge}$ and $\mathrm{Mn}_{3} \mathrm{Sn}$, New J. Phys. 19, 015008 (2017).

[4] K. Kuroda et al., Evidence for magnetic Weyl fermions in a correlated metal, Nat. Mater. 16, 1090 (2017). 
[5] J.-X. Yin et al., Giant and anisotropic spin-orbit tunability in a strongly correlated kagome magnet, Nature (London) 562, 91 (2018).

[6] S. Seiro, L. Jiao, , S. Kirchner, S. Hartmann, S. Friedemann, C. Krellner, C. Geibel, Q. Si, F. Steglich, and S. Wirth, Evolution of the Kondo lattice and non-Fermi liquid excitations in a heavy-fermion metal, Nat. Commun. 9, 3324 (2018).

[7] M. M. Yee et al., Imaging the Kondo insulating gap on $\mathrm{SmB}_{6}$, arXiv:1308.1085.

[8] L. Ye et al., Massive Dirac fermions in a ferromagnetic kagome metal, Nature (London) 555, 638 (2018).

[9] J.-X. Yin et al., Negative flat band magnetism in a spin-orbit-coupled correlated kagome magnet, Nat. Phys. 15, 443 (2019).

[10] N. Morali, R. Batabyal, P. K. Nag, E. Liu, Q. Xu, Y. Sun, B. Yan, C. Felser, N. Avraham, and H. Beidenkopf, Fermiarc diversity on surface terminations of the magnetic Weyl semimetal $\mathrm{Co}_{3} \mathrm{Sn}_{2} \mathrm{~S}_{2}$, Science 365, 1286 (2019).

[11] M. Ikhlas, T. Tomita, T. Koretsune, M.-T. Suzuki, D. Nishio-Hamane, R. Arita, , Y. Otani, and S. Nakatsuji, Large anomalous Nernst effect at room temperature in a chiral antiferromagnet, Nat. Phys. 13, 1085 (2017).

[12] H.-H. Yang, C.-C. Lee, Y. YoshidaM. Ikhlas, T. Tomita, A. Nugroho, T. Ozaki, S. Nakatsuji, and Y. Hasegawa, , Scanning tunneling microscopy on cleaved $\mathrm{Mn}_{3} \mathrm{Sn}(0001)$ surface, Sci. Rep. 9, 9677 (2019).

[13] Z. Lin et al., Flatbands and Emergent Ferromagnetic Ordering in $\mathrm{Fe}_{3} \mathrm{Sn}_{2}$ Kagome Lattices, Phys. Rev. Lett. 121, 096401 (2018).

[14] P. J. Brown, V. Nunez, F. Tasset, J. B. Forsyth, and P. Radhakrishna, Determination of the magnetic structure of $\mathrm{Mn}_{3} \mathrm{Sn}$ using generalized neutron polarization analysis, J. Phys. Condens. Matter 2, 9409 (1990).

[15] L. Jiao, Q. Xu, Y. Cheon, Y. Sun, C. Felser, E. Liu, and S. Wirth, Signatures for half-metallicity and nontrivial surface states in the kagome lattice Weyl semimetal $\mathrm{Co}_{3} \mathrm{Sn}_{2} \mathrm{~S}_{2}$, Phys. Rev. B 99, 245158 (2019).

[16] E. Krén et al., Study of the magnetic phase transformation in the $\mathrm{Mn}_{3} \mathrm{Sn}$ phase, Phys. Rev. B 80, 226 (1975).

[17] L. Jiao, S. Rößler, D. Kasinathan, P. F. S. Rosa, C. Guo, H. Yuan, C.-X. Liu, Z. Fisk, F. Steglich, and S. Wirth, Magnetic and defect probes of the $\mathrm{SmB}_{6}$ surface state, Sci. Adv. 4, eaau4886 (2018).

[18] M. Maltseva, M. Dzero, and P. Coleman, Electron Cotunneling into a Kondo Lattice, Phys. Rev. Lett. 103, 206402 (2009).

[19] L. Luo, C. Zhang, Y.-H. Jiang, and C. R. Brouwer, Emergence of Kondo lattice behavior in a van der Waals itinerant ferromagnet, $\mathrm{Fe}_{3} \mathrm{GeTe}_{2}$, Sci. Adv. 4, e1701799 (2018).

[20] Y. F. Yang, Fano effect in the point contact spectroscopy of heavy-electron materials, Phys. Rev. B 79, 1 (2009).

[21] P. Aynajian, E. H. da Silva Neto, C. V. Parker, Y. Huang, A. Pasupathy, J. Mydosh, and A. Yazdani, Visualizing the formation of the Kondo lattice and the hidden order in $\mathrm{URu}_{2} \mathrm{Si}_{2}$, Proc. Natl. Acad. Sci. U.S.A. 107, 10383 (2010).

[22] S. Ernst, S. Kirchner, C. Krellner, C. Geibel, G. Zwicknagl, F. Steglich, and S. Wirth, Emerging local Kondo screening and spatial coherence in the heavy-fermion metal $\mathrm{YbRh}_{2} \mathrm{Si}_{2}$, Nature (London) 474, 362 (2011).
[23] K. Nagaoka, T. Jamneala, M. Grobis, and M. F. Crommie, Temperature Dependence of a Single Kondo Impurity, Phys. Rev. Lett. 88, 077205 (2002).

[24] See Supplemental Material at http://link.aps.org/ supplemental/10.1103/PhysRevLett.125.046401 for details, which includes Refs. [25-43].

[25] P. E. Blöchl, Projector augmented-wave method, Phys. Rev. B 50, 17953 (1994).

[26] G. Kresse and D. Joubert, From ultrasoft pseudopotentials to the projector augmented-wave method, Phys. Rev. B 59, 1758 (1999).

[27] G. Kresse and J. Furthmüller, Efficiency of ab-initio total energy calculations for metals and semiconductors using a plane-wave basis set, Comput. Mater. Sci. 6, 15 (1996).

[28] J. P. Perdew, K. Burke, and M. Ernzerhof, Generalized Gradient Approximation Made Simple, Phys. Rev. Lett. 77, 3865 (1996).

[29] P. J. Brown, V. Nunez, F. Tasset, J. B. Forsyth, and P. Radhakrishna, Determination of the magnetic structure of $\mathrm{Mn}_{3} \mathrm{Sn}$ using generalized neutron polarization analysis, J. Phys. Condens. Matter 2, 9409 (1990).

[30] D. E. P. Vanpoucke and G. Brocks, Formation of Pt-induced $\mathrm{Ge}$ atomic nanowires on $\mathrm{Pt} / \mathrm{Ge}(001)$ : A density functional theory study, Phys. Rev. B 77, 241308 (2008).

[31] A. A. Mostofi, J. R. Yates, Y.-S. Lee, I. Souza, D. Vanderbilt, and N. Marzari, wannier90: A tool for obtaining maximally-localised Wannier functions, Comput. Phys. Commun. 178, 685 (2008).

[32] B. Möller and P. Wölfle, Magnetic order in the periodic Anderson model, Phys. Rev. B 48, 10320 (1993).

[33] R. Jullien and R. M. Martin, Ground-state and low-lying excitations of the periodic Anderson Hamiltonian in one dimension from finite-cell calculations, Phys. Rev. B 26, 6173 (1982).

[34] P. Sinjukow and W. Nolting, Exact mapping of periodic Anderson model to Kondo lattice model, Phys. Rev. B 65, 212303 (2002).

[35] K. Ueda, H. Tsunetsugu, and M. Sigrist, Singlet Ground State of the Periodic Anderson Model at Half Filling: A Rigorous Result, Phys. Rev. Lett. 68, 1030 (1992).

[36] R. Doradziński and J. Spałek, Mean-field magnetic phase diagram of the periodic Anderson model with the Kondocompensated phases, Phys. Rev. B 58, 3293 (1998).

[37] T. M. Rice and K. Ueda, Gutzwiller Variational Approximation to the Heavy-Fermion Ground State of the Periodic Anderson Model, Phys. Rev. Lett. 55, 995 (1985).

[38] H. Kaga, H. Kubo, and T. Fujiwara, Coherent Kondo-lattice state and the crossover transitions in the Anderson-lattice model, Phys. Rev. B 37, 341 (1988).

[39] M. Tachiki and S. Maekawa, Superconductivity in the Kondo lattice, Phys. Rev. B 29, 2497 (1984).

[40] M. Jarrell, Symmetric periodic Anderson model in infinite dimensions, Phys. Rev. B 51, 7429 (1995).

[41] G. D. Mahan, Many-Particle Physics (Springer, New York, 1990).

[42] K. Kang and B. I. Min, Equation-of-motion treatment of the impurity Anderson model with a finite on-site Coulomb repulsion, Phys. Rev. B 52, 10689 (1995).

[43] A. C. Hewson, The Kondo Problem to Heavy Fermions (Cambridge University Press, Cambridge, England, 1993). 
[44] T. A. Costi, Kondo Effect in a Magnetic Field and the Magnetoresistivity of Kondo Alloys, Phys. Rev. Lett. 85, 1504 (2000).

[45] J. E. Moore and X. G. Wen, Anomalous Magnetic Splitting of the Kondo Resonance, Phys. Rev. Lett. 85, 1722 (2000).
[46] P. Coleman, Introduction to Many-Body Physics (Cambridge University Press, Cambridge, England, 2015).

[47] J Hopkinson and P. Coleman, $\mathrm{LiV}_{2} \mathrm{O}_{4}$ : Frustration Induced Heavy Fermion Metal, Phys. Rev. Lett. 89, 267201 (2002). 